\title{
Multiparty quantum secret sharing of secure direct communication
}

\author{
Zhan-jun Zhang ${ }^{1,2}$ \\ ${ }^{1}$ Wuhan Institute of Physics and Mathematics, Chinese Academy of Sciences, Wuhan 430071, China \\ 2 School of Physics \& Material Science, Anhui University, Hefei 230039, China \\ Email: zhangzj@wipm.ac.cn
}

(Dated: July 9, 2018)

\begin{abstract}
Based on the two-step protocol [Phys. Rev. A68(03)042317], we propose a $(n, n)$-threshold multiparty quantum secret sharing protocol of secure direct communication. In our protocol only all the sharers collaborate can the sender's secure direct communication message be extracted. We show a variant version of this protocol based on the variant two-step protocol. This variant version can considerably reduce the realization difficulty in experiment. In contrast to the use of multi-particle GHZ states in the case that the sharer number is larger than 3, the use and identification of Bell states are enough in our two protocols disregarding completely the sharer number, hence, our protocols are more feasible in technique.
\end{abstract}

PACS: 03.67.Dd, 03.65.Ta, 89.70.+c

Quantum key distribution (QKD) is an ingenious application of quantum mechanics, in which two remote legitimate users (Alice and Bob) establish a shared secret key through the transmission of quantum signals and thereafter they can use this key to encrypt the secret messages or decrypt the encrypted messages. With quantum mechanics, other cryptographic task can be realized, such as quantum secret sharing (QSS). QSS is a generalization of classical secret sharing[1,2] to a quantum scenario [3], where the sender's secret message is distributed via quantum mechanical method among $n$ sharers in such a way that only all the sharers cooperate can they recover the secret message. The QSS is likely to play a key role in protecting secret quantum information, e.g., in secure operations of distributed quantum computation, sharing difficult-to-construct ancilla states and joint sharing of quantum money [6], and so on. Hence, after the pioneering QSS work proposed by Hillery et al. using three-particle and four-particle GHZ states [3], this kind of works on QSS attracted a great deal of attentions in both theoretical and experimental aspects [4-16,27]. All these works [3-16] can be divided into two kinds, one only deals with the QSS of classical messages (i.e., bits) [5-6,8-11,13-14,27], or only deals with the QSS of quantum information $[4,7,12,15-16]$ where the secret is an arbitrary unknown state in a qubit; and the other [3] studies both, that is, deals with QSS of classical messages and QSS of quantum information simultaneously. In all these works[3-16,27], when the party number is greater than 3, multi-particle GHZ states are used with only two exceptions[13,27]. Note that, so far all these works dealing with the QSS of classical messages (bits) allow in essence a sender to establish a joint key with receivers.

On the other hand, a novel concept, quantum secure direct communication (QSDC) has been proposed and pursued recently[17-20]. Different from QKD whose object is to distribute a common key between the two remote legitimate users of communication, QSDC can transit the secret messages directly without creating first a key to encrypt them. Hence, QSDC may be important in some applications which have been shown by Bostrom et al.[18] and Deng et al.[19-20].

Our one basic purpose in this paper is to generalize the concept of usual multiparty quantum 
secret sharing of key to the counterpart concept of multiparty quantum secret sharing of secure direct communication. As same as the advantages of QSDCs over QKDs, the advantages of such a generalization are intuitive. Based on a QSDC protocol (i.e., the two-step protocol) proposed by Deng et al.[19] using Einstein-Podolsky-Rosen (EPR) photon pair block, in this paper we will propose a multiparty quantum secret sharing protocol of secure direct communication. In our this protocol, only all the sharers collaborate can the secure direct communication message be obtained by all the sharers. Moreover, we will show a variant version of this protocol based on the variant two-step protocol we will propose. This variant version can considerably reduce the realization difficulty in experiment. In contrast to the use of multi-particle GHZ states in the case that the sharer number is larger than 3, the use and identification of Bell states are enough in our two protocols disregarding completely the sharer number, hence, our protocols are more feasible in technique. One will see these later.

Now let us turn to our multiparty quantum secret sharing (QSS) protocol of secure direct communication. For convenience, let us first describe a three-party protocol. Suppose Alice has a (2N)-bit secret message. She wants to send this secret message to two distant sharers, Bob and Charlie. One of them, Bob or Charlie, is not entirely trusted by Alice, and she knows that if the two guys coexist, the honest one will keep the dishonest one from doing any damages. The two sharers can infer the secret message only by their mutual assistance. Our following three-party QSS protocol of secure direct communication can achieve this goal with 7 steps.

(a) All the parties (Alice and the two sharers Bob and Charlie) agree on that each of the four Bell states can carry a two-bit classical message, i.e., $\phi^{+}, \phi^{-}, \psi^{+}$and $\psi^{-}$correspond to 00, 01, 10 and 11 , respectively. Here the four Bell states are defined as follows:

$$
\begin{aligned}
& \phi^{+}=(|0\rangle|0\rangle+|1\rangle|1\rangle) / \sqrt{2}=(|H\rangle|H\rangle+|V\rangle|V\rangle) / \sqrt{2}, \\
& \phi^{-}=(|0\rangle|0\rangle-|1\rangle|1\rangle) / \sqrt{2}=(|H\rangle|V\rangle+|V\rangle|H\rangle) / \sqrt{2}, \\
& \psi^{+}=(|1\rangle|0\rangle+|0\rangle|1\rangle) / \sqrt{2}=(|H\rangle|H\rangle-|V\rangle|V\rangle) / \sqrt{2}, \\
& \psi^{-}=(|1\rangle|0\rangle-|0\rangle|1\rangle) / \sqrt{2}=(|V\rangle|H\rangle-|H\rangle|V\rangle) / \sqrt{2},
\end{aligned}
$$

where $|0\rangle$ and $|1\rangle$ are the up and down eigenstates of $\sigma_{z},|H\rangle=(|0\rangle+|1\rangle) / \sqrt{2}$ and $|V\rangle=(|0\rangle-|1\rangle) / \sqrt{2}$ are the up and down eigenstates of $\sigma_{x}$. For convenience, $\{|0\rangle,|1\rangle\}$ is refereed to as the diagonal basis and $\{|H\rangle,|V\rangle\}$ the rectilinear basis hereafter.

(b) First Alice prepares an ordered $N$ EPR photon pair sequence according to her secret message. Then she prepares $k+j$ EPR photon pairs each randomly in one of the four Bell states. We call these photon pairs as checking photon pairs. Alice randomly inserts each of the $k+j$ checking photon pairs one by one into the ordered $N$ photon pair sequence and records each position. We denote the ordered $N+k+j$ EPR pair sequence with $\left[\left(X_{1}, Y_{1}\right),\left(X_{2}, Y_{2}\right), \ldots,\left(X_{N+k+j}, Y_{N+k+j}\right)\right]$. Here the subscript indicates the pair order in the sequence, and $X$ and $Y$ represent the two photons, respectively.

(c) Alice takes one photon from each EPR pair to form an ordered photon sequence, say, $\left[Y_{1}, Y_{2}, \ldots, Y_{N+k+j}\right]$. It is called the $Y$ sequence. The remaining EPR partner photons form another ordered sequence $\left[X_{1}, X_{2}, \ldots, X_{N+k+j}\right]$. It is called the $X$ sequence. Alice sends the $Y$ sequence to Bob. 
(d) After he receives the $Y$ sequence, for each photon in the sequence Bob randomly chooses a local unitary operation from $U_{1}, U_{2}, U_{3}, U_{4}$ and $H$ and performs this operation on it. After his encryptions (i.e., the local unitary operations), Bob sends the ordered $Y$ photons to Charlie. Here

$$
\begin{gathered}
U_{1}=|0\rangle\langle 0|+| 1\rangle\langle 1|=I, \\
U_{2}=|0\rangle\langle 0|-| 1\rangle\langle 1|=\sigma_{z}, \\
U_{3}=|0\rangle\langle 1|+| 1\rangle\langle 0|=\sigma_{x}, \\
U_{4}=|0\rangle\langle 1|-| 1\rangle\langle 0|=i \sigma_{y}, \\
H=(|0\rangle\langle 0|-| 1\rangle\langle 1|+| 0\rangle\langle 1|+| 1\rangle\langle 0|) / \sqrt{2}=\left(U_{2}+U_{3}\right) / \sqrt{2}=\left(\sigma_{z}+\sigma_{x}\right) / \sqrt{2},
\end{gathered}
$$

where $H$ is the so-called Hadamard operator. Define

$$
\begin{gathered}
\xi \equiv\left(\phi^{-}+\psi^{+}\right) / \sqrt{2}=(|H\rangle|0\rangle-|V\rangle|1\rangle) / \sqrt{2}=(|0\rangle|H\rangle+|1\rangle|V\rangle) / \sqrt{2}, \\
\eta \equiv\left(\phi^{+}-\psi^{-}\right) / \sqrt{2}=(|V\rangle|0\rangle+|H\rangle|1\rangle) / \sqrt{2}=(|0\rangle|H\rangle-|1\rangle|V\rangle) / \sqrt{2}, \\
\chi \equiv\left(\psi^{-}+\phi^{+}\right) / \sqrt{2}=(|H\rangle|0\rangle-|V\rangle|1\rangle) / \sqrt{2}=(|0\rangle|V\rangle+|1\rangle|H\rangle) / \sqrt{2}, \\
\zeta \equiv\left(\psi^{+}-\phi^{-}\right) / \sqrt{2}=(-|V\rangle|0\rangle+|H\rangle|1\rangle) / \sqrt{2}=(-|0\rangle|V\rangle+|1\rangle|H\rangle) / \sqrt{2} .
\end{gathered}
$$

For convenience, $\left\{\phi^{+}, \phi^{-}, \psi^{+}, \psi^{-}\right\}$is refereed to as the Bell state set and $\{\xi, \eta, \chi, \zeta\}$ the rotation state set hereafter. The nice feature of the $U_{i}(i=1,4)$ operation is that it transforms the state in both state sets (cf., Table 1 and Table 2). In contrast, the nice feature of $H$ is that it can realize the state transformation between the two sets (cf., Table 1 and Table 2). The purpose of choosing a set of five unitary operations is to protect the channel between Alice and Bob from Charlie's interception. For example, if Bob chooses randomly a unitary operation from only $U_{i}(i=1,4)$, Charlie could intercept the channel between Alice and Bob and replaces the $Y$ sequence by a fake one. Then when Bob sends the encrypted fake $Y$ sequence to Charlie, Charlie can know Bob's encryptions (i.e., his $U_{i}(i=1,4)$ operations) by Bell-state measurements on his fake pair sequence. Since Charlie has already had all the information about Bob's encryptions, he can perform the same encryptions as Bob did on his intercepted $Y$ sequence and accordingly he can readily retrieve the complete message without Bob's help.

Table 1 Results of four Bell states after the $U_{1}^{Y}, U_{2}^{Y}, U_{3}^{Y}, U_{4}^{Y}$ and $H_{Y}$ unitary operations. See text

\begin{tabular}{ccccc}
\multicolumn{5}{c}{ for details. } \\
\hline & $\phi_{X Y}^{+}$ & $\phi_{X Y}^{-}$ & $\psi_{X Y}^{+}$ & $\psi_{X Y}^{-}$ \\
\hline$U_{1}^{Y}$ & $\phi_{X Y}^{+}$ & $\phi_{X Y}^{-}$ & $\psi_{X Y}^{+}$ & $\psi_{X Y}^{-}$ \\
$U_{2}^{Y}$ & $\phi_{X Y}^{-}$ & $\phi_{X Y}^{+}$ & $\psi_{X Y}^{-}$ & $\psi_{X Y}^{+}$ \\
$U_{3}^{Y}$ & $\psi_{X Y}^{+}$ & $-\psi_{X Y}^{-}$ & $\phi_{X Y}^{+}$ & $-\phi_{X Y}^{-}$ \\
$U_{4}^{Y}$ & $\psi_{X Y}^{-}$ & $-\psi_{X Y}^{+}$ & $\phi_{X Y}^{-}$ & $-\phi_{X Y}^{+}$ \\
$H_{Y}$ & $\xi_{X Y}$ & $\eta_{X Y}$ & $\chi_{X Y}$ & $\zeta_{X Y}$ \\
\hline
\end{tabular}


Table 2 Results of the $\xi_{X Y}, \eta_{X Y}, \chi_{X Y}$ and $\zeta_{X Y}$ states after the $U_{1}^{Y}, U_{2}^{Y}, U_{3}^{Y}, U_{4}^{Y}$ and $H_{Y}$ unitary operations. See text for details.

\begin{tabular}{ccccc}
\hline & $\xi_{X Y}$ & $\eta_{X Y}$ & $\chi_{X Y}$ & $\zeta_{X Y}$ \\
\hline$U_{1}^{Y}$ & $\xi_{X Y}$ & $\eta_{X Y}$ & $\chi_{X Y}$ & $\zeta_{X Y}$ \\
$U_{2}^{Y}$ & $\chi_{X Y}$ & $-\zeta_{X Y}$ & $\xi_{X Y}$ & $-\eta_{X Y}$ \\
$U_{3}^{Y}$ & $\eta_{X Y}$ & $\xi_{X Y}$ & $\zeta_{X Y}$ & $\chi_{X Y}$ \\
$U_{4}^{Y}$ & $-\zeta_{X Y}$ & $\chi_{X Y}$ & $-\eta_{X Y}$ & $\xi_{X Y}$ \\
$H_{Y}$ & $\phi_{X Y}^{+}$ & $\phi_{X Y}^{-}$ & $\psi_{X Y}^{+}$ & $\psi_{X Y}^{-}$ \\
\hline
\end{tabular}

(e) After confirming that Bob has received an ordered $Y$ sequence, Alice publicly announces the positions of $k$ checking photon pairs. For each of these $k$ checking photon pairs, Alice first lets Charlie measure the corresponding checking photon in his lab by using the diagonal basis or the rectilinear basis and publish his measurement outcome. Alice's choice of letting Charlie use which basis is completely random. Then Alice lets Bob publish which unitary operation he has performed on the checking photon when it passes by him. If Bob's unitary operation is $H$, then Alice measures the corresponding checking photon in her lab by using the different basis from the one she let Charlie use. Otherwise, she measures the photon using the same basis as Charlie used. By the way, if only one basis is used, then Eve can steal secret information without being detected. Since only Alice knows the initial state of each checking photon pair, after all her measurements, she can determine the error rate according to the correlations (cf., equations 1-4 and 10-13). If the error rate exceeds the threshold, the process is aborted. Otherwise, the process continues and Alice sends the retained $N+j$ ordered sequence to Charlie.

(f) After Charlie receives the photons, if Bob and Charlie collaborate, both Bob and Charlie can obtain a raw secret message including Alice's secret message and additional $2 j$ useless bits. The detailed procedure is as follows. For each photon pair, if Bob's unitary operation is $U_{i}(i=1,4)$, then Bob and Charlie directly perform a Bell-state measurement on the photon pair. If Bob's unitary operation is $H$, then they first perform a Hardamard operation on the $Y$ photon and then perform a Bell-state measurement on the photon pair. According to their measurement outcome and Bob's exact unitary operation they can deduce the two secret bits Alice imposed on this photon pair. For example, without loss of generality, suppose their outcome is $\psi^{-}$. If Bob's unitary operation is $U_{3}$, then according to Table 1 they can infer that the secret bits Alice imposed on this photon pair is '01' corresponding to the initial state $\phi^{-}$; if Bob's unitary operation is $H$, they can infer that the secret bits Alice imposed on this photon pair is '11' corresponding to the initial state $\psi^{-}$. On the other hand, if Bob and Charlie do not collaborate, then neither of them can get access to Alice's secret message with $100 \%$ certainty.

(g) Alice publicly announces the initial states and positions of the retained $j$ checking pairs for Bob and Charlie to extract her exact secret message from the raw secret message and to check whether the $N+j \mathrm{X}$ photons travelling from Alice site to Charlie's site have been attacked, which is called message authentification. Even if the photons are attacked, the eavesdropper Eve can not get access to any useful information but interrupt the transmissions quantum channel between Alice and the last receiver (Charlie).

So far we have proposed the three-party QSS protocol of secure direct communication based on Deng et al.'s two-step QSDC protocol[19] by using the EPR photon pair block. Its security proof is similar to those in Refs.[18-19,21-23] with entangled photons. The proof of our this protocol is based on the 
security for the transmission of the $\mathrm{Y}$ sequence. This is very similar to the security for the transmission of the $\mathrm{C}$ sequence in the two-step protocol. If Alice, Bob and Charlie could not detect eavesdropper Eve in the transmission of the Y sequence, the eavesdropper would adopt the intercept-resend strategy to eavesdrop the quantum channel and easily reads out the secure direct communication message. In fact, in our protocol Alice, Bob and Charlie can know whether the transmission of the $\mathrm{Y}$ sequence is secure or not by using the checking procedure based on the qubit correlation of each Bell state and Eve will be found out if she eavesdrops the quantum channel. Moreover, even in a worse case that there is an insider (Bob or Charlie) who wants to occupy independently the secure direct communication without another sharer's help and thereby eavesdrops the quantum line, this insider's eavesdropping can be also detected by using the detecting method. Once the security for the transmission of the Y sequence is not ensured, Alice will abort the transmission of the $\mathrm{X}$ sequence, thereby no information will be leaked to the eavesdropper. As same as in the two-step protocol, the transmission and the security check of the $\mathrm{Y}$ sequence in our this protocol is also very similar to the procedure in BBM92 QKD protocol[26], where one qubit in an EPR pair is sent to a party and another is sent to another party. Here the X sequence is retained securely in Alice's lab and Eve (or an insider) can not get access to it at all before Alice's eavesdropping detection. Hence, the security of transmission of the Y sequence can be simply reduced to the security of the BBM92 QKD protocol. The proof of security for BBM92 in ideal and practical conditions has been given so far[21,23]. Hence our this protocol is also unconditionally secure.

Now let us generalize the three-party QSS protocol of secure direct communication to a $n$-party $(n \geq 4)$ QSS one. Suppose that Alice is the message sender who would like to send a secret massage to Bob, Charlie, Dick, ..., York and Zach (there is totally $n-1$ sharers). Alice does not trust anyone but the sharer entirety. She hopes that, only the sharer entirety cooperates can her secure direct communication message be obtained by the entirety, otherwise, no one of the entirety can get access to her secret message with $100 \%$. Our $n$-party $(n \geq 4)$ QSS protocol of secure direct communication can achieve this goal. The first four step and the last step of the $n$-party $(n \geq 4)$ QSS protocol of secure direct communication is the same as these in the three-party QSS protocol. Therefore, we describe the $n$-party $(n \geq 4)$ QSS protocol from the step 5 .

(V) After receiving the $\mathrm{Y}$ sequence, Charlie encrypts it in the same way as Bob and then sends it to the next sharer, say, Dick. Similar procedures are repeated until the $(n-1)$ th sharer Zach receives the Y sequence.

(VI) After confirming that Zach has received the ordered $Y$ sequence, Alice publicly announces the positions of $k$ checking photon pairs. For each of these $k$ checking photon pairs, Alice first lets Zach measure the corresponding checking photon in his lab by using either the diagonal basis or the rectilinear basis and publish his measurement outcome. Alice's choice of letting Zach use which basis is completely random. Then Alice lets all other sharers without Zach publish which unitary operations they have performed on the checking photon when it passes by them. Since only Alice knows the initial state of each checking photon pair, using Table 1 and Table 2 she can works out its final state before measurements. If the final state belongs to the rotation state set, then Alice measures the corresponding checking photon in her lab by using the different basis from the one she has let Zach use. Otherwise, she measures the photon using the same basis as she has let Zach use. Similar procedures are repeated until all actions including Zach's measurements and publishes, all other sharers' publishes of unitary operations and Alice's unitary operations and measurements are completed for the $k$ checking photon pairs. Then Alice can determine the error rate in terms of the correlations (cf., equations 1-4 and 10-13). If the error rate exceeds the threshold, the process is 
aborted. Otherwise, the process continues and Alice sends the retained $(N+j)$ ordered sequence to Zach.

(VII) After Zach receives the photons, if all the sharers collaborate, then they can obtain a raw secret message including Alice's secret message and additional $2 j$ useless bits. The detailed procedure is as follows. For each photon pair, they the unitary operation $U=U_{B}^{+} U_{C}^{+} U_{D}^{+} \ldots U_{Z}^{+}$on the $\mathrm{Y}$ photon, where $U_{B}\left(U_{C}, U_{D}, \ldots, U_{Z}\right)$ represents the unitary operation Bob (Charlie, Dick, ..., York, Zach) has ever performed on the $\mathrm{Y}$ photon. After this unitary operation, the state of this photon pair is recovered to its initial state, thereby all the sharers can take a Bell-state measurement to extract Alice's two-bit secret information. After all the photon pairs are dealt with, they can obtain the raw secret message. On the other hand, if anyone of all the sharers does not collaborate, then none of them can get access to Alice's secret message with $100 \%$ certainty.

(VIII) As mentioned previously, the present last step is same as the counterpart step in the threeparty QSS protocol of secure direct communication. This step ensures all the sharers to extract Alice's exact secret message from the raw secret message and the security of transmission of the $(N+j) \mathrm{X}$ photons.

So far we have established a $n$-party QSS protocol of secure direct communication. Its security is the same as the security of three-party QSS protocol of secure direct communication, which is also unconditionally secure.

In our multiparty QSS protocol of secure direct communication, Alice is the preparer of the EPR photon pair sequence and the $\mathrm{X}$ and $\mathrm{Y}$ photon sequences are sent from her by two-steps. Such unilateral transmissions determines that when all sharers extract Alice's secret message they should perform reversal unitary operations to recover the state of each relative pair to its initial state. It is known that the more operations the more difficulties in experiment. In the following we will take advantage of the change of transmission to reduce the difficulty in experiment. One will see this later. Suppose Alice has a $2 N$-bit secret message. She would like to send a secret massage to Bob, Charlie, Dick, ..., York and Zach (there is totally $n-1$ sharers). Alice does not trust anyone but the entirety. She hopes that, only the entirety cooperate can her secure direct communication message be obtained by the entirety, otherwise, no one of the entirety can get access to her secret message with $100 \%$. The variant version of our this $n$-party QSS protocol of secure direct communication is depicted as follows.

(1) All the parties (Alice and all the sharers Bob, Charlie, Dick, ..., York and Zach) agree on that each of the four Bell states can carry a two-bit classical message, i.e., $\phi^{+}, \phi^{-}, \psi^{+}$and $\psi^{-}$correspond to $00,01,10$ and 11 , respectively.

(2) Alice lets Zach prepare an ordered $N+k+j$ EPR pair block and initial states of all the pairs are in $\phi^{+}$. We denote the ordered $N+k+j$ EPR pair sequence is denoted with $\left[\left(X_{1}, Y_{1}\right),\left(X_{2}, Y_{2}\right), \ldots,\left(X_{N+k+j}, Y_{N+k+j}\right)\right]$.

(3) Zach takes one photon from each EPR pair to form an ordered photon sequence, say, $\left[Y_{1}, Y_{2}, \ldots, Y_{N+k+j}\right]$. It is called the $Y$ sequence. The remaining EPR partner photons form another ordered sequence $\left[X_{1}, X_{2}, \ldots, X_{N+k+j}\right]$. It is called the $X$ sequence. Alice sends the $Y$ sequence to the $(n-2)$ th sharer York.

(4) After he receives the $Y$ sequence, for each photon in the sequence York randomly chooses a local unitary operation from $U_{1}, U_{2}, U_{3}, U_{4}$ and $H$ and performs this operation on it and then sends it to the $(n-3)$ th sharer. The $(n-3)$ th sharer encrypts the $\mathrm{Y}$ sequence in the same way as York and then sends it to the next sharer, an so on. Similar procedures are repeated until the first sharer Bob finishes his encryptions. Bob sends the Y sequence to Alice.

(5) Alice selects randomly $k$ photons and publicly announces their positions in the Y sequence. Alice 
lets Zach measure the partner photons in the $\mathrm{X}$ sequence of the selected photons in the $\mathrm{Y}$ sequence either in the diagonal basis or in the rectilinear basis. To prevent any sharer's intercept-resend attack, for each selected photon, Alice randomly selects a sharer one by one and let him or her tell her this sharer's message till she obtains all sharer's messages. Here Zach's message includes which basis he has used to measure the partner photon and his measurement outcome, York's (,.., Charlie's, Bob's) message is which unitary operation he has ever performed on the selected photon. By the way, such random choice is to protect the quantum channel from any sharer's interception. Hence, Alice can know via calculation the exact final state of her selected photon should be after Zach's measurement on the partner photon, and thereby after her measurements she can determine the error rate. If the error rate exceeds the threshold, the process is aborted. Otherwise, the process continues and Alice performs unitary operations on the retained photons in the $\mathrm{Y}$ sequence to encode a raw secret message including her secret messages and additional $2 j$ useless bits. Alice sends these encoded photons to Zach.

(6) After Zach receives the photons, if all the sharers collaborate, then they can obtain a raw secret message including Alice's secret message and additional $2 j$ useless bits. The detailed procedure is as follows. For each photon pair, they can work out the exact state before Alice's encoding. If it belongs to the Bell state set, then they directly take a Bell-state measurement and thereby they can know the two bits Alice has ever imposed on this pair. Otherwise, they first perform a Hadamard operation on the Y photon and take a Bell-state measurement. From Table 2 they can infer the state of this pair before the Hadamard operation and thereby they can know the two bits Alice has ever imposed on the pair. Note that after the change of the transmission direction the reversal operations are unnecessary anymore during the raw message extraction. To this sense, we think this variant version has considerably reduced the realization difficulty in experiment.

(7) Alice publicly announces positions of the additional $j$ pairs and her encodings for all the sharers to extract her exact secret message from the raw secret message and to check whether the $(N+$ j) Y photons travelling from Alice site to Charlie's site have been attacked. Even if the photons are attacked, the eavesdropper Eve can not get access to any useful information but interrupt the transmissions quantum channel between Alice and Zach.

So far we have presented the variant version of our multiparty QSS protocol of secure direct communication. It is based on a variant two-step protocol we just proposed. Different from the first multiparty QSS protocol where the sender Alice sends the $\mathrm{Y}$ and $\mathrm{X}$ sequence by two steps and her secret message is encoded on the $\mathrm{X}$ sequence, in the variant version it is the last sharer Zach who sends the $\mathrm{Y}$ sequence to fetch the secret message. Such a change of transmissions ensures the realization difficulty in experiment can be considerably reduced. As mentioned previously, its security proof is also very similar these in Refs.[18-19, 21-23] and can be reduced to the proof of the BBM92 QKD protocol[26]. Hence, it is also unconditionally secure.

In all our present protocols, the multi-particle GHZ states in all other existing multiparty QSS schemes are not necessary. Although in [15] it is claimed that only Bell states are needed, the identification of multi-particle GHZ state is still necessary. In our multiparty SSQI protocol, only the use and identification of Bell states are needed. Till now to our knowledge only five-photon GHZ state is realized in experiment[24], more-photon GHZ state is still desired in experiment. Hence, in the case when the sharer number is large enough, the usual QSS schemes can only be possible in theory. In contrast, for any large number of sharers, our protocols can securely work provided the use and identification of Bell states are possible. Hence, the present multiparty QSS protocols are more feasible in technique[25]. 
To summarize, in this paper based on the two-step protocol we have presented a multiparty quantum secret sharing protocol of secure direct communication. In our protocol only all the sharers collaborate can the sender's secure direct communication messages be extracted. We show a variant version of this protocol based on the variant two-step protocol. This variant version can considerably reduce the realization difficulty in experiment. In contrast to the use of multi-particle GHZ states in the case that the sharer number is larger than 3, the use and identification of Bell states are enough in our two protocols disregarding completely the sharer number, hence, our protocols are more feasible in technique.

\section{Acknowledgement}

This work is supported by the National Natural Science Foundation of China under Grant No. 10304022 .

\section{References}

[1] B. Schneier, Applied Cryptography (Wiley, New York, 1996) p. 70.

[2] J. Gruska, Foundations of Computing (Thomson Computer Press, London, 1997) p. 504.

[3] M. Hillery, V. Buzk, and A. Berthiaume, Phys. Rev. A 59, 1829 (1999).

[4] R. Cleve, D. Gottesman, and H. K. Lo, Phys. Rev. Lett. 83, 648 (1999).

[5] A. Karlsson, M. Koashi, and N. Imoto, Phys. Rev. A 59, 162 (1999).

[6] D. Gottesman, Phys. Rev. A 61, 042311 (2000).

[7] S. Bandyopadhyay, Phys. Rev. A 62, 012308 (2000).

[8] W. Tittel, H. Zbinden, and N. Gisin, Phys. Rev. A 63, 042301 (2001).

[9] V. Karimipour and A. Bahraminasab, Phys. Rev. A 65, 042320 (2002).

[10] H. F. Chau, Phys. Rev. A 66, 060302 (2002).

[11] S. Bagherinezhad and V. Karimipour, Phys. Rev. A 67, 044302 (2003).

[12] L. Y. Hsu, Phys. Rev. A 68, 022306 (2003).

[13] G. P. Guo and G. C. Guo, Phys. Lett. A 310, 247 (2003).

[14] L. Xiao, G. L. Long, F. G. Deng, and J. W. Pan, Phys. Rev. A 69, 052307 (2004).

[15] Y. M. Li, K. S. Zhang, and K. C. Peng, Phys. Lett. A 324, 420 (2004).

[16] A. M. Lance, T. Symul, W. P. Bowen, B. C. Sanders, and P. K. Lam, Phys. Rev. Lett. 92, 177903 (2004).

[17] A. Beige, B. G. Englert, C. Kurtsiefer, and H.Weinfurter, Acta Phys. Pol. A 101, 357 (2002).

[18] K. Bostrom K and F. Felbinger. Phys. Rev. Lett. 89, 187902 (2002).

[19] F. G. Deng, G. L. Long, and X. S. Liu, Phys. Rev. A 68, 042317 (2003).

[20] F. G. Deng and G. L. Long, Phys. Rev. A 69, 052319 (2004).

[21] H. Inamori, L. Rallan, and V. Verdral, J. Phy. A 34, 6913 (2001).

[22] G. L. Long and X. S. Liu, Phys. Rev. A 65, 032302 (2002) .

[23] E. Waks, A. Zeevi, and Y. Yamamoto, Phys. Rev. A 65, 052310 (2002).

[24] Z. Zhao, Y. A. Chen, A. N. Zhang, T. Yang, H. J. Briegel, and J. W. Pan, Nature (London) 430, 54 (2004).

[25] Y. H. Kim, S. P. Kulik, and Y. Shih, Phys. Rev. Lett. 86, 1370 (2001).

[26] C. H. Bennett, G. Brassard, and N. D. Mermin, Phys. Rev. Lett. 68, 557 (1992).

[27] Z. J. Zhang, Y. Li, and Z. X. Man, resubmitted to Phys. Rev. A 